\title{
Evidence for unidimensional low-energy excitations as the origin of persistent spin dynamics in geometrically frustrated magnets
}

\author{
A. Yaouanc,,${ }^{1,2,3}$ P. Dalmas de Réotier, ${ }^{1,2}$ A. Bertin, ${ }^{1,2}$ C. Marin,,${ }^{1,2}$ E. Lhotel, ${ }^{4}$ A. Amato, ${ }^{3}$ and C. Baines ${ }^{3}$ \\ ${ }^{1}$ Université Grenoble Alpes, INAC-SPSMS, F-38000 Grenoble, France \\ ${ }^{2}$ CEA, INAC-SPSMS, F-38000 Grenoble, France \\ ${ }^{3}$ Laboratory for Muon-Spin Spectroscopy, Paul Scherrer Institute, CH-5232 Villigen-PSI, Switzerland \\ ${ }^{4}$ Institut Néel, CNRS and Université Joseph Fourier, BP 166, F-38042 Grenoble Cedex 9, France
}

(Received 20 November 2013; revised manuscript received 23 December 2014; published 27 March 2015)

\begin{abstract}
We report specific heat, magnetic, and muon spin relaxation measurements performed on a polycrystalline sample of the normal spinel $\mathrm{CdHo}_{2} \mathrm{~S}_{4}$. The rare-earth ions sit on a lattice of corner-sharing regular tetrahedra as in pyrochlore compounds. Magnetic ordering is detected at $T_{\mathrm{c}} \simeq 0.87 \mathrm{~K}$. From spin-lattice relaxation rate measurements on both sides of $T_{\mathrm{c}}$ we uncover similar magnetic excitation modes driving the so-called persistent spin dynamics at $T<T_{\mathrm{c}}$. Unidimensional excitations are argued to be at its origin. Often observed spin loop structures are suggested to support these excitations. The possibility of a generic mechanism for their existence is discussed.
\end{abstract}

DOI: 10.1103/PhysRevB.91.104427

PACS number(s): 75.40.-s, 76.75.+i, 75.25.-j

Magnetic materials with coupled spins located on cornersharing tetrahedra are expected to exhibit geometrical magnetic frustration because their spatial arrangements are such that they prevent the simultaneous minimization of all the interaction energies. Typical examples are given by the pyrochlore insulator compounds of generic chemical formula $R_{2} M_{2} \mathrm{O}_{7}$, where $R$ is a rare-earth ion and $M$ a nonmagnetic element [1]. For instance, $\mathrm{Ho}_{2} \mathrm{Ti}_{2} \mathrm{O}_{7}$, for which the net interaction between the spins is ferromagnetic, has been the first recognized spinice system and an analogy has been drawn between the proton positions in common ice $I_{h}$ and the spin configuration [2]. Its properties seem mostly described by classical physics. On the other hand, $\mathrm{Yb}_{2} \mathrm{Ti}_{2} \mathrm{O}_{7}$ is an example of a three-dimensional quantum spin liquid [3], at least for the best available sample characterized by a clear specific heat peak at its first-order transition $[4,5]$. While this transition is reminiscent of that observed between the gas and liquid states of conventional matter, it is characterized by a strong quantum entanglement [6]. As a last example, we cite $\mathrm{Yb}_{2} \mathrm{Sn}_{2} \mathrm{O}_{7}$, which is a splayed ferromagnet, i.e., essentially a ferromagnetic compound [7], with an emergent gauge field [8].

Although this physics is exotic, an interpretation at the mean-field level is within reach [8]. However, the most exotic property of these compounds lies in their dynamics. The origin of the ubiquitous persistent spin dynamics observed in geometrically frustrated magnetic materials is still elusive. Its most famous signature is a finite and approximately temperatureindependent spin-lattice relaxation rate $\lambda_{Z}$ observed in these compounds below $\approx 1 \mathrm{~K}$ irrespective of the presence of a magnetic order or not. Conventionally, at temperatures well below $\left|\theta_{\mathrm{CW}}\right|$ where $\theta_{\mathrm{CW}}$ is the Curie-Weiss temperature, a magnetic system should order and $\lambda_{Z}$ should vanish when the temperature approaches zero. Still, a finite and temperatureindependent $\lambda_{Z}$ is found in the ordered state of magnetic compounds such as $\mathrm{Cu}_{2} \mathrm{Cl}(\mathrm{OH})_{3}$ [9], $\mathrm{Gd}_{2} \mathrm{Sn}_{2} \mathrm{O}_{7}$ [10,11], and $\mathrm{Gd}_{2} \mathrm{Ti}_{2} \mathrm{O}_{7}$ [12], to cite few reported cases. Also surprising is the absence of muon spin spontaneous precession in muon spin relaxation $(\mu \mathrm{SR})$ measurements below the magnetic critical temperature $T_{\mathrm{c}}$ for $\mathrm{Tb}_{2} \mathrm{Sn}_{2} \mathrm{O}_{7}$ [13,14], $\mathrm{Er}_{2} \mathrm{Ti}_{2} \mathrm{O}_{7}$ [15,16], and
$\mathrm{Yb}_{2} \mathrm{Sn}_{2} \mathrm{O}_{7}$ [7]. This lack of spontaneous precession stems from unexpected excitation modes below $T_{\mathrm{c}}$, as first shown for $\mathrm{Tb}_{2} \mathrm{Sn}_{2} \mathrm{O}_{7}[13,17,18]$. A physical mechanism accounting for them is still missing.

Recently normal spinels of chemical formula $\mathrm{Cd} R_{2} X_{4}$ $(X=\mathrm{S}, \mathrm{Se})$ have attracted some attention [19]. In this crystal structure, the $R$ ions form the same lattice of cornersharing regular tetrahedra as in the pyrochlore compounds; see Fig. 1(a). A spin-ice behavior has been discovered for $\mathrm{CdEr}_{2} \mathrm{Se}_{4}$ [20], highlighting the interest of extending the number of compounds with geometrical frustration on a three-dimensional lattice.

Here we report bulk and $\mu$ SR measurements for the normal thiospinel $\mathrm{CdHo}_{2} \mathrm{~S}_{4}$. We find evidence for a magnetic phase transition with similar magnetic excitation modes on both sides of $T_{\mathrm{c}}$. We show that these modes are at the origin of the observed persistent spin dynamics as fingerprinted by $\lambda_{Z}$.

The synthesis of $\mathrm{CdHo}_{2} \mathrm{~S}_{4}$ powder has followed a two-step route. First, $\mathrm{Ho}_{2} \mathrm{~S}_{3}$ has been prepared starting with holmium metal $(4 \mathrm{~N})$ and sulfur $(5 \mathrm{~N})$ properly mixed and heat treated in a vacuum-sealed quartz tube up to $720^{\circ} \mathrm{C}$ over 2 weeks, the temperature being increased step by step to avoid excessive pressure due to sulfur vapor. The phase has been checked by $\mathrm{x}$-ray powder diffraction. Secondly, $\mathrm{Ho}_{2} \mathrm{~S}_{3}$ has been mixed with commercial $\mathrm{CdS}$ powder $(5 \mathrm{~N})$ and pressed under 4 tons into 13-mm-diameter pellets to improve solid-state reaction. A heat treatment of the resulting product has been achieved up to $900^{\circ} \mathrm{C}$ for 2 weeks in a sealed quartz tube under vacuum. The final yellow/brown ceramic has been found to be the $\mathrm{CdHo}_{2} \mathrm{~S}_{4}$ phase perfectly crystallized without any x-ray detected foreign phases. Finally, sintered pellets were obtained from this ceramic after grinding and compaction with the same press, followed by a heat treatment at $600^{\circ} \mathrm{C}$ over $6 \mathrm{~h}$ under vacuum.

The investigation of the macroscopic properties has consisted of measurements of the heat capacity using a Physical Property Measurement System (Quantum Design, Inc.), and of the magnetization and ac susceptibility. These magnetization experiments have been performed by the extraction method 
(a)

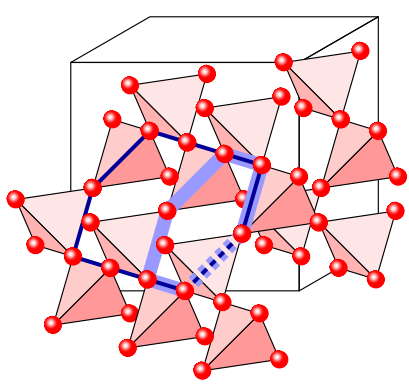

FIG. 1. (Color online) (a) Rare-earth ions lattice in the pyrochlore $R_{2} M_{2} \mathrm{O}_{7}$ and normal spinel $\mathrm{Cd} R_{2} X_{4}$ compounds. The thicker light blue (thinner dark blue) bold line represents a 6 (10)-site loop. (b) Low-temperature heat capacity of $\mathrm{CdHo}_{2} \mathrm{~S}_{4}$.

using a Magnetic Property Measurement System (Quantum Design, Inc.) for measurements down to $2 \mathrm{~K}$ and a superconducting quantum interference device magnetometer developed at the Institut Néel [21] for measurements down to $0.07 \mathrm{~K}$ and up to an external magnetic field $B_{\text {ext }}=8 \mathrm{~T}$.

The $\mu$ SR experiments were carried out at the Swiss Muon Source ( $\mathrm{S} \mu \mathrm{S}$, Paul Scherrer Institute, Switzerland) either at the Low Temperature Facility (LTF) or the General Purpose Surface-Muon instrument (GPS), depending on the temperature range. Measurements were performed with the transverse (longitudinal) geometry in which the external field defining the $Z$ axis of a referential frame is applied perpendicular (parallel) to the initial muon spin polarization. The measured physical quantity is the so-called $\mu \mathrm{SR}$ asymmetry time spectrum, which describes the evolution of the projection of the muon polarization perpendicular to (along) the direction of the initial polarization [22]. The spectrum is denoted as $a_{0} P_{X}^{\exp }(t)$ $\left[a_{0} P_{Z}^{\exp }(t)\right]$ for the transverse (longitudinal) field geometry. Zero applied field measurements were also performed in the longitudinal geometry.

The heat capacity $C_{\mathrm{p}}$ depicted in Fig. 1(b) displays a fairly narrow peak at $T_{\mathrm{c}} \simeq 0.86 \mathrm{~K}$, signaling a thermodynamic phase transition, and a broad hump centered at about $3 \mathrm{~K}$. This latter feature, attributed to the onset of short-range magnetic correlations and not described by the Landau free energy, is commonly observed in frustrated magnets [23].

We now consider the bulk magnetic measurements. Figure 2 depicts the inverse of the susceptibility, i.e., $1 / \chi$, versus temperature in a broad temperature range. The Curie-Weiss law provides a good description of $\chi$ above $150 \mathrm{~K}$ with $\theta_{\mathrm{CW}}=$ $-3.6(5) \mathrm{K}$ and the so-called paramagnetic moment $m_{\mathrm{pm}}=$ $10.8(7) \mu_{\mathrm{B}}$. This latter number compares favorably with the isolated $\mathrm{Ho}^{3+}$ value, $m_{\mathrm{eff}}=10.6 \mu_{\mathrm{B}}$. From measurements with $B_{\text {ext }}=0.1 \mathrm{~T}$, Lau et al. have reported a similar value for $m_{\mathrm{pm}}$, but a larger $\theta_{\mathrm{CW}}$ absolute value $\left[\theta_{\mathrm{CW}}=-7.6(2) \mathrm{K}\right][19]$. Because $\theta_{\mathrm{CW}}$ is negative, the dominant exchange interactions are antiferromagnetic. A slight deviation from the Curie-Weiss law is observed below $150 \mathrm{~K}$.

In Fig. 3, the variation of $\chi$ at low temperature is displayed: it exhibits a weak maximum at $T_{\mathrm{c}} \simeq 0.88 \mathrm{~K}$. This maximum

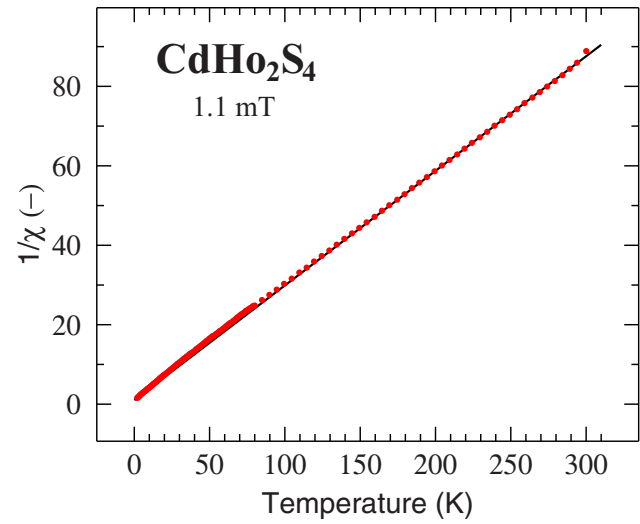

FIG. 2. (Color online) Inverse of the magnetic susceptibility versus temperature. The data have been measured in a magnetic field of $1.1 \mathrm{mT}$. The solid line results from a fit of the Curie-Weiss law to the data measured above $150 \mathrm{~K}$. The susceptibility is dimensionless since we use SI units.

is somewhat stronger in ac susceptibility data (not shown) recorded in the 5-100 Hz frequency range. Together with the aforementioned $C_{\mathrm{p}}$ peak, these results point to a magnetic transition at $T_{\mathrm{c}}$. An uprise in $\chi(T)$ is detected well below $T_{\mathrm{c}}$. An origin for it could be the presence of residual free spins in our sample. Modeling this upturn as explained in the caption of Fig. 3, a very good fit is obtained for a $1.7 \%$ fraction of $\mathrm{Ho}^{3+}$ ions being in a paramagnetic state with a moment $m_{\text {eff }}$; see full line in Fig. 3. The Curie-Weiss temperature associated with this fraction of the spins is negligible: $\theta_{\mathrm{CW}, \mathrm{fs}}=-37(1) \mathrm{mK}$.

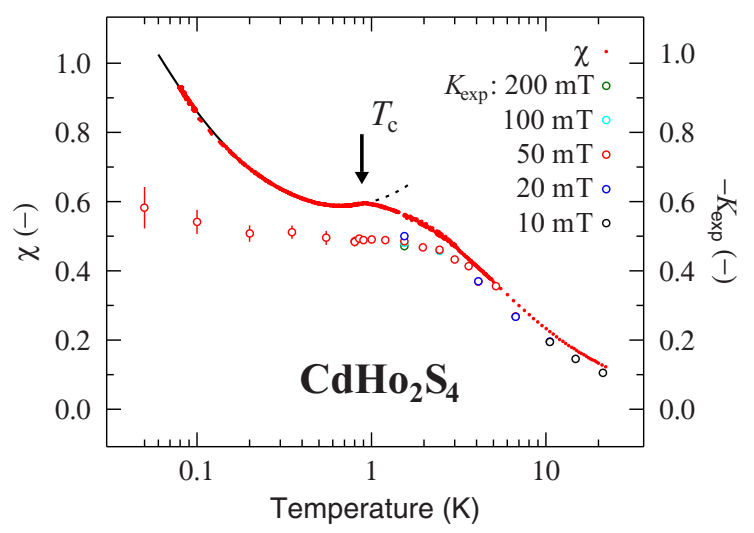

FIG. 3. (Color online) Magnetic measurements for a $\mathrm{CdHo}_{2} \mathrm{~S}_{4}$ powder sample. The data for the magnetic susceptibility $\chi$ versus temperature were recorded with external fields $B_{\text {ext }}$ ranging from 0.5 to $50 \mathrm{mT}$ and were found to match one another. The field was applied in the plane of the sample pellet so that the demagnetization field is small. The solid line at low temperature together with the dotted line extension above $T_{\mathrm{c}}$ represents a fit of the function $C_{\mathrm{fs}} /\left(T-\theta_{\mathrm{CW}, \mathrm{fs}}\right)+$ $a+b T$ to the data recorded below $T_{\mathrm{c}}$. The former term represents the contribution of weakly interacting $\mathrm{Ho}^{3+}$ spins, while the remaining terms describe the majority spin state; see main text. The quantity $K_{\text {exp }}$, defined in the main text and measured for different $B_{\text {ext }}$ as indicated in the figure, is shown for comparison. 


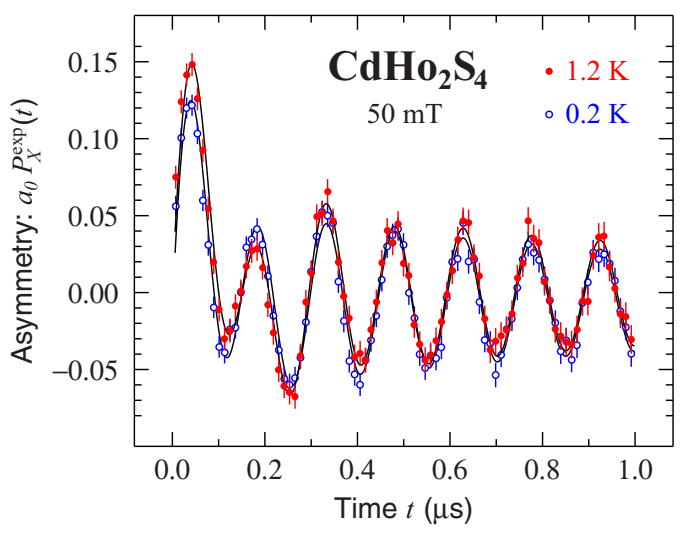

FIG. 4. (Color online) $\mu$ SR spectra recorded in a transverse of $50 \mathrm{mT}$ at 0.2 and $1.2 \mathrm{~K}$. The full lines are the results of fits to the data. The model consists of a sum of two exponentially damped cosine functions, the former and latter accounting for muons stopped in the sample and its surroundings, respectively. The amplitudes extracted from the fits are temperature independent for the two components. The precession frequency $v_{\mu}$ of the former provides a measure of the mean field $\left\langle B_{\mu}\right\rangle=2 \pi v_{\mu} / \gamma_{\mu}$ at the muon site in the sample; see main text for details. $\gamma_{\mu}=851.615 \mathrm{Mrads}^{-1} \mathrm{~T}^{-1}$ is the muon gyromagnetic ratio.

In order to check the hypothesis that the $\chi$ upturn arises from a small fraction of the $\mathrm{Ho}^{3+}$ spins, we have used the $\mu$ SR technique in the transverse-field geometry. Here, a field $\mathbf{B}_{\text {ext }}$ transverse to the initial polarization of the muon beam is applied to the sample. The muon Larmor precession is then monitored (Fig. 4), yielding the mean-field magnitude $\left\langle B_{\mu}\right\rangle$ at the muon site which uniformly probes the sample volume. The quantity of interest here is $K_{\text {exp }}=\left(\left\langle B_{\mu}\right\rangle-B_{\text {ext }}\right) / B_{\text {ext }}$. Once corrected for the demagnetization and Lorentz fields, ${ }^{1}$ this quantity is proportional to a microscopic susceptibility which can be compared to the macroscopic susceptibility discussed above. The proportionality coefficient depends on the muonsystem coupling. In Fig. $3,-K_{\exp }(T)$ is found to track $\chi(T)$ for $T>3 \mathrm{~K}$. However, for $T \lesssim T_{\mathrm{c}},-K_{\exp }$ only weakly depends on the temperature. This observation confirms that the uprise of $\chi(T)$ is not representative of the vast majority of the $\mathrm{Ho}^{3+}$ spins in $\mathrm{CdHo}_{2} \mathrm{~S}_{4}$.

The measurements of the magnetic moment per holmium ion as a function of field at $4.2 \mathrm{~K}$ and down to $0.07 \mathrm{~K}$ are presented in Fig. 5. The moment tends to saturation with $m_{\mathrm{sat}} \simeq 8.2 \mu_{\mathrm{B}} / \mathrm{Ho}^{3+}$ under $8 \mathrm{~T}$, fairly consistent with $m_{\text {sat }} \simeq 7.5 \mu_{\mathrm{B}} / \mathrm{Ho}^{3+}$ at $5 \mathrm{~T}$ previously reported [19]. The value for $m_{\text {sat }}$ is much larger in $\mathrm{CdHo}_{2} \mathrm{~S}_{4}$ than in $\mathrm{Ho}_{2} \mathrm{Ti}_{2} \mathrm{O}_{7}$ [24], suggesting a different type of anisotropy in the two

\footnotetext{
${ }^{1}$ According to Eq. (5.66) of Ref. [22], the local susceptibility $K_{\mu}$ is related to $K_{\exp }$ through the relation $K_{\mu}=K_{\exp }-\left(1 / 3-N^{Z}\right) \chi$, which can be applied since the thin-pellet-shaped sample can be approximated to a strongly oblate ellipsoid of revolution. The relevant demagnetization coefficient $N^{Z}$ being definitively larger than $1 / 3$, the demagnetization and Lorentz field corrections for $-K_{\exp }$ will result in a downward shift in Fig. 3, proportional to $\chi$, which rules out any low-temperature uprise.
}

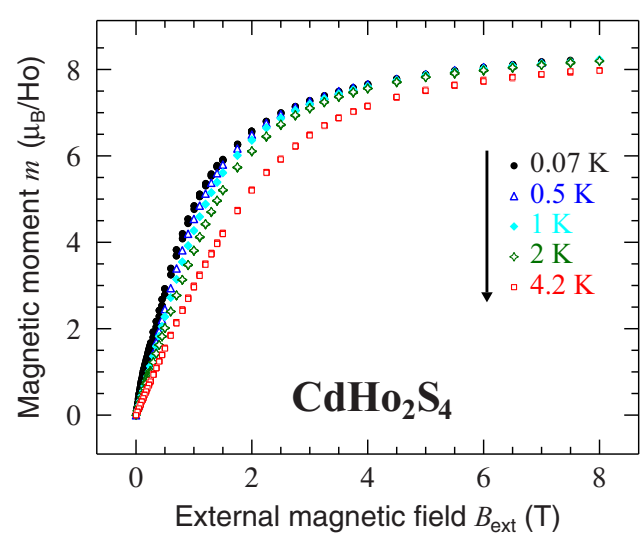

FIG. 5. (Color online) Magnetic moment per holmium ion of a $\mathrm{CdHo}_{2} \mathrm{~S}_{4}$ powder versus field measured for different temperatures as indicated in the figure.

compounds. With the presently available experimental data, the electronic configuration of the $\mathrm{Ho}^{3+}$ spins, in particular, their crystal electric field energy levels and wave functions, cannot be discussed further. In Fig. 6 we display the derivative of the magnetic moment value with respect to the external field. At 4.2 and $2 \mathrm{~K}$ it monotonically decays as the external field is increased. At lower temperatures the derivative passes through a maximum at a field of approximately half a tesla. This maximum is the signature of a metamagneticlike behavior, as usually observed in antiferromagnets. The sharp decrease of the derivative for fields up to $\simeq 0.2 \mathrm{~T}$ at $0.07 \mathrm{~K}$ is more surprising. While we have no definitive explanation for it at the moment, it could be associated with the fraction of free spins detected in the susceptibility measurements.

We now discuss the zero-field $\mu$ SR spectra. Examples of spectra recorded on both sides of $T_{\mathrm{c}}$ are displayed in Fig. 7 . Contrary to expectation for an ordered magnet, no spontaneous oscillation is detected below $T_{c}$. There is also no missing asymmetry which would result from an unresolved oscillation. We simply find an exponential-like relaxation on each side of

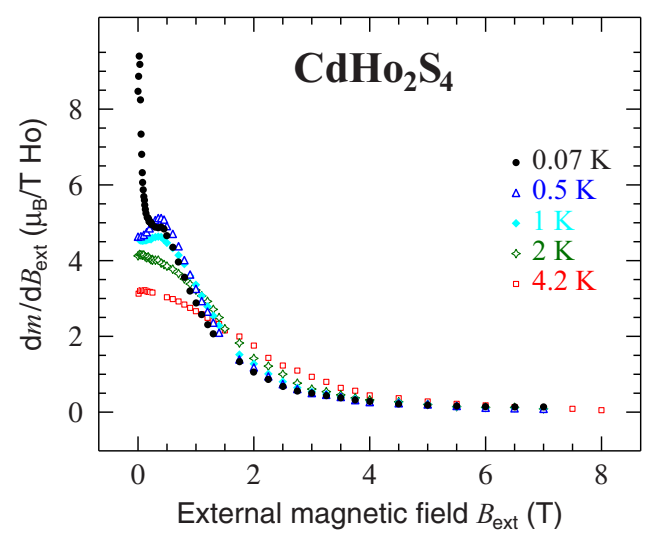

FIG. 6. (Color online) External field derivative of the $\mathrm{Ho}^{3+}$ magnetic moment of a $\mathrm{CdHo}_{2} \mathrm{~S}_{4}$ powder for different temperatures as indicated in the figure. 


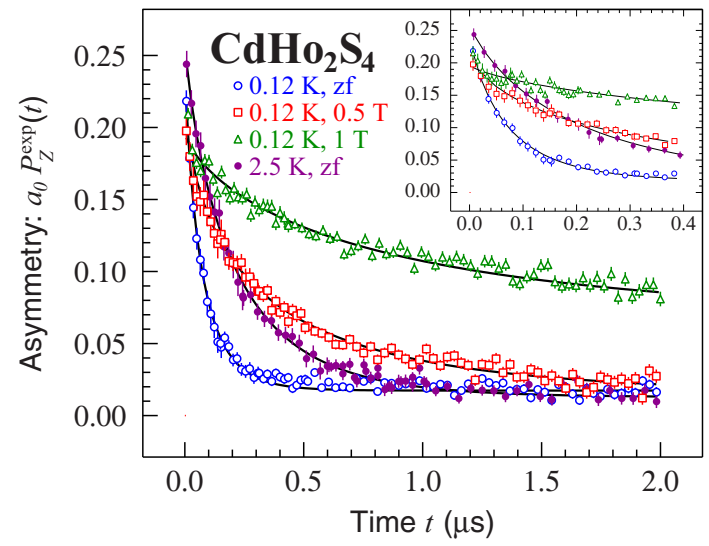

FIG. 7. (Color online) Four $\mu \mathrm{SR}$ spectra recorded for a $\mathrm{CdHo}_{2} \mathrm{~S}_{4}$ powder sample, two zero-field spectra taken on both sides of the magnetic phase transition temperature $T_{\mathrm{c}}$, and two longitudinal field spectra recorded at $T=0.12 \mathrm{~K}$, i.e., $T \ll T_{\mathrm{c}}$. The early time details are shown in the insert. The solid lines result from fits as explained in the main text.

$T_{c}$. This is a signature of a strong dynamical spin component below $T_{\mathrm{c}}$.

The function $a_{0} P_{Z}^{\exp }(t)=a_{\mathrm{s}} P_{Z}(t)+a_{\mathrm{bg}}$, where the second time-independent component accounts for the muons missing the sample, has been fitted to the spectra. A good description of $P_{Z}(t)$ in zero field is obtained with a stretched exponential relaxation, i.e., $P_{Z}(t)=\exp \left[-\left(\lambda_{Z} t\right)^{\beta}\right]$. The exponent $\beta$ has been found constant with $\beta=0.8$ up to $0.6 \mathrm{~K}$, and then it increases steadily with temperature, reaching $\beta=1$ above $10 \mathrm{~K}$. As seen in the insert of Fig. 8, to the critical temperature corresponds a faint anomaly in $\lambda_{Z}(T)$. The rate $\lambda_{Z}$ is finite and becomes almost temperature indepen-

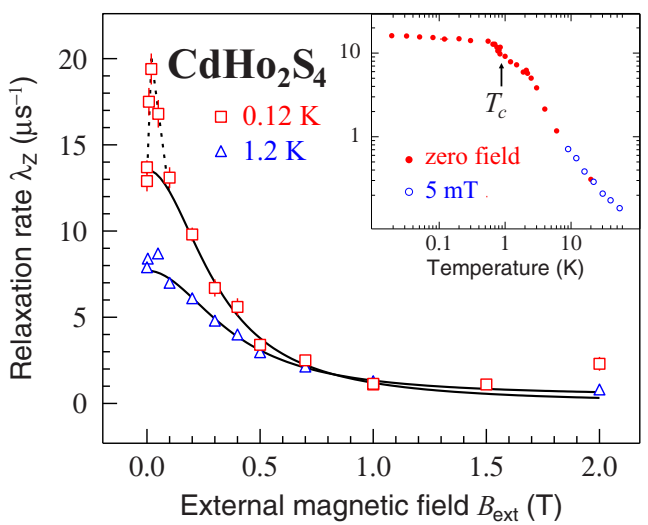

FIG. 8. (Color online) Spin-lattice relaxation rate $\lambda_{Z}$ versus longitudinal field intensity $B_{\text {ext }}$ and temperature $T$ for a $\mathrm{CdHo}_{2} \mathrm{~S}_{4}$ powder. In the main frame is displayed $\lambda_{Z}\left(B_{\text {ext }}\right)$ for two temperatures below and above $T_{\mathrm{c}}$. The $\lambda_{Z}(T=0.12 \mathrm{~K})$ maximum occurs at $20 \mathrm{mT}$. The solid lines are explained in the main text. The dashed line at small $B_{\text {ext }}$ and $T=0.12 \mathrm{~K}$ is a guide to the eyes. In the insert is displayed $\lambda_{Z}(T)$ measured from 0.019 to $55 \mathrm{~K}$ under zero field or $B_{\text {ext }}=5 \mathrm{mT}$. The temperature $T_{\mathrm{c}}$ at which the compound exhibits a magnetic phase transition is specified by an arrow. dent below $T_{\mathrm{c}}$, a signature of the so-called persistent spin dynamics.

The results for $\mathrm{CdHo}_{2} \mathrm{~S}_{4}$ are consistent with the previous observation for the ordered spin ice $\mathrm{Tb}_{2} \mathrm{Sn}_{2} \mathrm{O}_{7}$ [13], While a spontaneous oscillation is also absent in the order-bydisorder antiferromagnet $\mathrm{Er}_{2} \mathrm{Ti}_{2} \mathrm{O}_{7}[15,16]$ and the splayed ferromagnet $\mathrm{Yb}_{2} \mathrm{Sn}_{2} \mathrm{O}_{7}$ [7], their relaxation below $T_{c}$ is not exponential-like. For $\mathrm{Tb}_{2} \mathrm{Sn}_{2} \mathrm{O}_{7}$ and $\mathrm{CdHo}_{2} \mathrm{~S}_{4}$ we are in fact in the fast fluctuation regime for which $\gamma_{\mu} \Delta_{\mathrm{rms}} \tau_{\mathrm{c}} \ll 1$, where $\Delta_{\mathrm{rms}}$ and $\tau_{\mathrm{c}}$ are the standard deviation of the field distribution at the muon site and the correlation time of the fieldcorrelation function, respectively. This key feature will enable us to investigate the relaxation in terms of spin-correlation functions. Before leaving this qualitative discussion, we note that an inflection point in $\lambda_{Z}(T)$ is present around $20 \mathrm{~K}$. It may correspond to a crossover from a crystal-electric-field excitation-dominated regime $[22,23]$ to a strongly correlated low-temperature paramagnetic regime. We also note that $\mathrm{Tb}_{2} \mathrm{Sn}_{2} \mathrm{O}_{7}$ has been discussed in terms of a partially ordered magnet owing to the coexistence of static and dynamical magnetic modes in its ordered phase [18]. Such a situation may apply for $\mathrm{CdHo}_{2} \mathrm{~S}_{4}$.

Figure 7 also displays two longitudinal-field spectra recorded at $0.12 \mathrm{~K}$. They are reasonably represented by a stretched exponential function. This means that the system is characterized by a distribution of relaxation rates [22,25-27]. In Fig. 8 we present $\lambda_{Z}\left(B_{\text {ext }}\right)$. While at $1.2 \mathrm{~K}$ it drops monotonically as $B_{\text {ext }}$ is increased, it exhibits a maximum at $\approx 20 \mathrm{mT}$ for $T=0.12 \mathrm{~K}$ before decreasing at larger $B_{\text {ext }}$. We also note a slight upturn in $\lambda_{Z}$ above $1.5 \mathrm{~T}$ which could be associated with a crystal-electric-field effect. A low-field $\lambda_{Z}$ maximum has already been reported [13,28-30]. An avoided level-crossing resonance might be at play [31]. A quantitative analysis should provide further information. Neglecting this maximum, we find $\lambda_{Z}\left(B_{\text {ext }}\right)$ to be well described by a conventional Lorentzian behavior (full lines in Fig. 8): $\lambda_{Z}\left(B_{\text {ext }}\right)=$ $2 \gamma_{\mu}^{2} \Delta_{\mathrm{rms}}^{2} \tau_{\mathrm{c}} /\left(1+\gamma_{\mu}^{2} B_{\mathrm{ext}}^{2} \tau_{\mathrm{c}}^{2}\right)[22,32]$. At $0.12 \mathrm{~K}$ a fit to the data gives $\tau_{\mathrm{c}}=3.8(3) \mathrm{ns}$ and $\Delta_{\mathrm{rms}}=49(4) \mathrm{mT}$, and at $1.2 \mathrm{~K}$, the parameters are $\tau_{\mathrm{c}}=3.08(8) \mathrm{ns}$ and $\Delta_{\mathrm{rms}}=40(1) \mathrm{mT}$. An additional small constant $\lambda_{Z, 0}=0.4 \mu \mathrm{s}^{-1}$ needs to be added to the Lorentzian at $1.2 \mathrm{~K}$. Surprisingly, the two parameters of the Lorentzian function have approximately the same values at 0.12 and $1.2 \mathrm{~K}$. This suggests the same type of excitations to be involved in the relaxation of the muon spin in the ordered and paramagnetic states. We also note that the paramagnetic fluctuation time scale of a few nanoseconds is anomalously long: from the energy scale given by the value of $\left|\Theta_{\mathrm{CW}}\right|$ one would expect a fluctuation time at least 2 orders of magnitude shorter.

Before discussing our experimental result in terms of intrinsic properties of the magnetic fluctuation modes that have been uncovered, we note that an alternative explanation has been proposed for the finite and temperature-independent relaxation rate measured at low temperature [33]. The model put forward by Quémerais and co-workers is based on the coherent diffusion of polaronic muons rather than magnetic fluctuations. However, this interpretation of the $\mathrm{Dy}_{2} \mathrm{Ti}_{2} \mathrm{O}_{7}$ data $[34,35]$ leads to a muon hopping rate nearly 3 orders of magnitude larger than that measured on the isostructural nonmagnetic material $\mathrm{Y}_{2} \mathrm{Ti}_{2} \mathrm{O}_{7}$ [36]. Moreover, spin dynamics 
has been detected in $\mathrm{Dy}_{2} \mathrm{Ti}_{2} \mathrm{O}_{7}$ down to $0.1 \mathrm{~K}$ [37]. Although no data is available concerning muon diffusion in $\mathrm{CdHo}_{2} \mathrm{~S}_{4}$, here we will not consider this possibility. Indeed, the similarity of the data in this material, in the pyrochlore systems listed at the beginning of this text, and in $\mathrm{Cu}_{2} \mathrm{Cl}(\mathrm{OH})_{3}$ strongly suggests that an explanation generic to three dimensional networks of corner-sharing tetrahedral spins must pertain.

To explain the low-temperature finite and approximately temperature-independent zero-field $\lambda_{Z}$ value, a Raman relaxation process involving two magnetic excitations has been put forward [12]. Generalizing this picture, we write

$$
\lambda_{Z}=\mathcal{C} \int_{\Delta}^{\infty} f\left[\epsilon /\left(k_{\mathrm{B}} T\right)\right] g_{\mathrm{m}}^{2}(\epsilon) \mathrm{d} \epsilon .
$$

Here $\mathcal{C}$ is a temperature-independent constant and $f(x)=$ $n(x)[1 \pm n(x)$, with $n(x)$ the Bose-Einstein or Fermi-Dirac distribution function and the + or - signs are for bosonic or fermionic excitations, respectively. We have introduced the magnetic density of states responsible for the relaxation $g_{\mathrm{m}}(\epsilon)$ and an energy gap $\Delta$. To get $\lambda_{Z}$ temperature independent, we need $g_{\mathrm{m}}(\epsilon)=b_{\mu} \epsilon^{-1 / 2}$ and $\left(\Delta-E_{\mathrm{F}}\right)$ or $(\Delta-\mu)$ proportional to temperature, i.e., equal to $a_{\mu} k_{\mathrm{B}} T$, where $a_{\mu}$ and $b_{\mu}$ are finite constants. We have denoted $E_{\mathrm{F}}$ the Fermi energy and $\mu$ the chemical potential (needed if the boson number is not fixed). The inverse square root form for $g_{\mathrm{m}}(\epsilon)$ needs to be verified only at low energy [12].

Expressing $g_{\mathrm{m}}(\epsilon)$ in terms of the spin correlation function $\left\langle\mathbf{J}_{\mathbf{q}}(t) \mathbf{J}_{-\mathbf{q}}(0)\right\rangle$, we obtain

$$
g_{\mathrm{m}}(\epsilon)=\sum_{\mathbf{q}} \int_{-\infty}^{\infty} \frac{\left\langle\mathbf{J}_{\mathbf{q}}(t) \cdot \mathbf{J}_{-\mathbf{q}}(0)\right\rangle}{\left\langle\mathbf{J}_{\mathbf{q}}(0) \cdot \mathbf{J}_{-\mathbf{q}}(0)\right\rangle} \exp \left(\frac{i \epsilon t}{\hbar}\right) \frac{\mathrm{d} t}{2 \pi \hbar} .
$$

The sum is over the first Brillouin zone vectors. We recall $\left\langle\mathbf{J}_{\mathbf{q}}(t) \cdot \mathbf{J}_{-\mathbf{q}}(0)\right\rangle=\sum_{i} \exp (-i \mathbf{q} \cdot \mathbf{i})\left\langle\mathbf{J}_{0}(t) \cdot \mathbf{J}_{i}(0)\right\rangle$, where $\mathbf{J}_{i}$ and $\mathbf{J}_{0}$ are the spins at the lattice point $i$ and at the origin of the lattice, respectively. Since muons probe the very-low-energy spin excitations, it is justified to consider the correlation function at long times. In this limit it is governed by a diffusion equation for a Heisenberg Hamiltonian system [38-40], i.e., $\left\langle\mathbf{J}_{0}(t) \cdot \mathbf{J}_{i}(0)\right\rangle \propto 1 /(D|t|)^{d / 2}$, where $d$ is the dimensionality of the spin system and $D$ a diffusion coefficient. Since the Fourier transform of $\sqrt{1 /|t|}$ is $\sqrt{2 \pi /|\omega|}=\sqrt{2 \pi \hbar /|\varepsilon|}$, unidimensional spin structures, i.e., $d=1$, are inferred to explain the low-temperature $\lambda_{Z}$ plateau found for geometrically frustrated magnetic materials, at least when the relaxation is exponential-like. Although the algebraic decay was originally derived from a phenomenological high-temperature theory for a Heisenberg system [38], a microscopic analysis leads to the same form for the correlations at low temperature in one-dimensional quantum spin systems with anisotropic exchange [41].

We tentatively associate the low-energy unidimensional excitations inferred from the temperature-independent spinlattice relaxation rate observed in numerous frustrated magnets to loop spin structures that have been considered theoretically (see, e.g., Refs. [42-44] for three-dimensional systems) and suggested from neutron-scattering experiments. A hexamer pattern was reported in the paramagnetic phase of the spinel chromite $A \mathrm{Cr}_{2} \mathrm{O}_{4}$ with $A=\mathrm{Zn}$ [45] and then for $A=\mathrm{Cd}$ [46], and later on for $A=\mathrm{Mg}$ in the paramagnetic and ordered magnetic states $[47,48]$. Results for the itinerant system $\mathrm{Y}_{0.97} \mathrm{Sc}_{0.03} \mathrm{Mn}_{2}$ display a broadly similar feature [49]. Hexamer correlations seem also present in the spinice system $\mathrm{Dy}_{2} \mathrm{Ti}_{2} \mathrm{O}_{7}$ [50]. An illustration for a hexamer structure is presented in Fig. 1(a), as well as another putative structure.

The similarity of $\lambda_{Z}\left(B_{\text {ext }}\right)$ measured for $\mathrm{CdHo}_{2} \mathrm{~S}_{4}$ on both sites of the transition shows unidimensional excitations to be present in both phases. The unusual long time scale observed for the spin dynamics is then consistent with the relatively large number of spins involved in the process. A possible origin for the drop of $\lambda_{Z}$ above $\approx 1 \mathrm{~K}$ might be excitation interactions which would break the spin-correlation power-law decay.

The ubiquitous low-temperature excitations that we infer suggest a generic mechanism. Emergent monopoles are the low-temperature magnetic excitations of spin-ice systems, i.e., of ferromagnetically interacting spins on a lattice of cornersharing tetrahedra [51,52]. An all-in all-out spin arrangement for the same lattice can be viewed as a lattice of magnetic octupoles [53]. Although still not proven, the same picture implying multipoles may apply to other antiferromagnetic structures. These poles interact through an effective, possibly screened, Coulomb interaction. We suggest to investigate whether this interaction could explain the excitations we have uncovered.

In summary, the thiospinel $\mathrm{CdHo}_{2} \mathrm{~S}_{4}$ in which the rareearth spins form a lattice of corner-sharing regular tetrahedra undergoes a magnetic transition at $T_{\mathrm{c}} \simeq 0.87 \mathrm{~K}$. A signature of anomalously slow fluctuation modes is found in the paramagnetic state. Similar modes are present in the ordered phase. Spin dynamics is observed down to at least $T_{\mathrm{c}} / 50$ through a finite and roughly temperature independent muon spin-lattice relaxation rate $\lambda_{Z}$. This is shown to be the signature of emergent unidimensional spin excitations. Since the $\lambda_{Z}$ plateau has been found in virtually all the frustrated compounds including the pyrochlore, kagome, and triangular systems, ${ }^{2}$ we conjecture the unidimensional spin excitations to be a generic feature of geometrically frustrated magnets, should the system order or not.

We thank C. Paulsen for the use of his SQUID dilution magnetometer. P.D.R. gratefully acknowledges partial support from Prof. H. Keller from the University of Zurich. This research project has been partially supported by the European Commission under the 7th Framework Programme through the Research Infrastructures action of the Capacities Programme, Contract No. CP-CSA_INFRA-2008-1.1.1, No. 226507-NMI3. Part of this work was performed at the Swiss Muon Source, Paul Scherrer Institute, Villigen, Switzerland.

\footnotetext{
${ }^{2}$ See, e.g., Refs. [9] and [12] for the lattice of corner-sharing tetrahedra, Ref. [54] for the kagome lattice, and Ref. [55] for the triangular lattice.
} 
[1] J. S. Gardner, M. J. P. Gingras, and J. E. Greedan, Rev. Mod. Phys. 82, 53 (2010).

[2] M. J. Harris, S. T. Bramwell, D. F. McMorrow, T. Zeiske, and K. W. Godfrey, Phys. Rev. Lett. 79, 2554 (1997).

[3] J. A. Hodges, P. Bonville, A. Forget, A. Yaouanc, P. Dalmas de Réotier, G. André, M. Rams, K. Królas, C. Ritter, P. C. M. Gubbens, C. T. Kaiser, P. J. C. King, and C. Baines, Phys. Rev. Lett. 88, 077204 (2002).

[4] A. Yaouanc, P. Dalmas de Réotier, C. Marin, and V. Glazkov, Phys. Rev. B 84, 172408 (2011).

[5] K. A. Ross, L. R. Yaraskavitch, M. Laver, J. S. Gardner, J. A. Quilliam, S. Meng, J. B. Kycia, D. K. Singh, T. Proffen, H. A. Dabkowska, and B. D. Gaulin, Phys. Rev. B 84, 174442 (2011).

[6] L. Balents, Nature (London) 464, 199 (2010).

[7] A. Yaouanc, P. Dalmas de Réotier, P. Bonville, J. A. Hodges, V. Glazkov, L. Keller, V. Sikolenko, M. Bartkowiak, A. Amato, C. Baines, P. J. C. King, P. C. M. Gubbens, and A. Forget, Phys. Rev. Lett. 110, 127207 (2013).

[8] L. Savary and L. Balents, Phys. Rev. Lett. 108, 037202 (2012).

[9] X. G. Zheng, H. Kubozono, K. Nishiyama, W. Higemoto, T. Kawae, A. Koda, and C. N. Xu, Phys. Rev. Lett. 95, 057201 (2005).

[10] P. Dalmas de Réotier, P. C. M. Gubbens, and A. Yaouanc, J. Phys.: Condens. Matter 16, S4687 (2004).

[11] Y. Chapuis, P. Dalmas de Réotier, C. Marin, A. Yaouanc, A. Forget, A. Amato, and C. Baines, Physica B 404, 686 (2009).

[12] A. Yaouanc, P. Dalmas de Réotier, V. Glazkov, C. Marin, P. Bonville, J. A. Hodges, P. C. M. Gubbens, S. Sakarya, and C. Baines, Phys. Rev. Lett. 95, 047203 (2005).

[13] P. Dalmas de Réotier, A. Yaouanc, L. Keller, A. Cervellino, B. Roessli, C. Baines, A. Forget, C. Vaju, P. C. M. Gubbens, A. Amato, and P. J. C. King, Phys. Rev. Lett. 96, 127202 (2006).

[14] F. Bert, P. Mendels, A. Olariu, N. Blanchard, G. Collin, A. Amato, C. Baines, and A. D. Hillier, Phys. Rev. Lett. 97, 117203 (2006).

[15] J. Lago, T. Lancaster, S. J. Blundell, S. T. Bramwell, F. L. Pratt, M. Shirai, and C. Baines, J. Phys.: Condens. Matter 17, 979 (2005).

[16] P. Dalmas de Réotier, A. Yaouanc, Y. Chapuis, S. H. Curnoe, B. Grenier, E. Ressouche, C. Marin, J. Lago, C. Baines, and S. R. Giblin, Phys. Rev. B 86, 104424 (2012).

[17] Y. Chapuis, A. Yaouanc, P. Dalmas de Réotier, S. Pouget, P. Fouquet, A. Cervellino, and A. Forget, J. Phys.: Condens. Matter 19, 446206 (2007).

[18] K. C. Rule, G. Ehlers, J. S. Gardner, Y. Qiu, E. Moskvin, K. Kiefer, and S. Gerischer, J. Phys.: Condens. Matter 21, 486005 (2009).

[19] G. C. Lau, R. S. Freitas, B. G. Ueland, P. Schiffer, and R. J. Cava, Phys. Rev. B 72, 054411 (2005).

[20] J. Lago, I. Živković, B. Z. Malkin, J. Rodriguez Fernandez, P. Ghigna, P. Dalmas de Réotier, A. Yaouanc, and T. Rojo, Phys. Rev. Lett. 104, 247203 (2010).

[21] C. Paulsen, in Introduction to Physical Techniques in Molecular Magnetism: Structural and Macroscopic Techniques - Yesa 1999, edited by F. Palacio, E. Ressouche, and J. Schweizer (Servicio de Publicaciones de la Universidad de Zaragoza, Zaragoza, 2001).
[22] A. Yaouanc and P. Dalmas de Réotier, Muon Spin Rotation, Relaxation, and Resonance: Applications to Condensed Matter (Oxford University Press, Oxford, 2011).

[23] P. Dalmas de Réotier, A. Yaouanc, P. C. M. Gubbens, C. T Kaiser, C. Baines, and P. J. C. King, Phys. Rev. Lett. 91, 167201 (2003).

[24] O. A. Petrenko, M. R. Lees, and G. Balakrishnan, Phys. Rev. B 68, 012406 (2003).

[25] C. P. Lindsey and G. D. Patterson, J. Chem. Phys. 73, 3348 (1980).

[26] M. N. Berderan-Santos, E. N. Bodunov, and B. Valeur, Chem. Phys. 315, 171 (2005).

[27] D. C. Johnston, Phys. Rev. B 74, 184430 (2006).

[28] A. Zorko, F. Bert, P. Mendels, P. Bordet, P. Lejay, and J. Robert, Phys. Rev. Lett. 100, 147201 (2008).

[29] A. Yaouanc, P. Dalmas de Réotier, Y. Chapuis, C. Marin, S. Vanishri, D. Aoki, B. Fåk, L. P. Regnault, C. Buisson, A. Amato, C. Baines, and A. D. Hillier, Phys. Rev. B 84, 184403 (2011).

[30] P. J. Baker, M. J. Matthews, S. R. Giblin, P. Schiffer, C. Baines, and D. Prabhakaran, Phys. Rev. B 86, 094424 (2012).

[31] A. Abragam, C. R. Acad. Sci. 299, 95 (1984).

[32] A. G. Redfield, IBM J. Res. Dev. 1, 19 (1957).

[33] P. Quémerais, P. McClarty, and R. Moessner, Phys. Rev. Lett. 109, 127601 (2012).

[34] J. Lago, S. J. Blundell, and C. Baines, J. Phys.: Condens. Matter 19, 326210 (2007).

[35] S. R. Dunsiger, A. A. Aczel, C. Arguello, H. Dabkowska, A. Dabkowski, M.-H. Du, T. Goko, B. Javanparast, T. Lin, F. L. Ning, H. M. L. Noad, D. J. Singh, T. J. Williams, Y. J. Uemura, M. J. P. Gingras, and G. M. Luke, Phys. Rev. Lett. 107, 207207 (2011).

[36] J. A. Rodriguez, A. Yaouanc, B. Barbara, E. Pomjakushina, P. Quémerais, and Z. Salman, Phys. Rev. B 87, 184427 (2013).

[37] J. S. Gardner, G. Ehlers, P. Fouquet, B. Farago, and J. R. Stewart, J. Phys.: Condens. Matter 23, 164220 (2011).

[38] N. Bloembergen, Physica 15, 386 (1949).

[39] L. Van Hove, Phys. Rev. 95, 1374 (1954).

[40] H. Benner and J. P. Boucher, in Magnetic Properties of Layered Transition Metal Compounds, edited by L. J. de Jongh (Kluwer Academic Publishers, Dordrecht, 1990), pp. 323-378.

[41] J. Sirker, R. G. Pereira, and I. Affleck, Phys. Rev. B 83, 035115 (2011).

[42] J. Villain, Z. Phys. B 33, 31 (1979).

[43] M. Hermele, M. P. A. Fisher, and L. Balents, Phys. Rev. B 69, 064404 (2004).

[44] R. G. Melko and M. J. P. Gingras, J. Phys.: Condens. Matter 16, R1277 (2004).

[45] S.-H. Lee, C. Broholm, W. Ratcliff, G. Gasparovic, Q. Huang, T. H. Kim, and S.-W. Cheong, Nature (London) 418, 856 (2002).

[46] J.-H. Chung, M. Matsuda, S.-H. Lee, K. Kakurai, H. Ueda, T. J. Sato, H. Takagi, K.-P. Hong, and S. Park, Phys. Rev. Lett. 95, 247204 (2005).

[47] K. Tomiyasu, H. Suzuki, M. Toki, S. Itoh, M. Matsuura, N. Aso, and K. Yamada, Phys. Rev. Lett. 101, 177401 (2008).

[48] K. Tomiyasu, T. Yokobori, Y. Kousaka, R. I. Bewley, T. Guidi, T. Watanabe, J. Akimitsu, and K. Yamada, Phys. Rev. Lett. 110, 077205 (2013).

[49] R. Ballou, E. Lelièvre-Berna, and B. Fåk, Phys. Rev. Lett. 76, 2125 (1996). 
[50] T. Yavors'kii, T. Fennell, M. J. P. Gingras, and S. T. Bramwell, Phys. Rev. Lett. 101, 037204 (2008).

[51] I. A. Ryzhkin, JETP 101, 481 (2005).

[52] C. Castelnovo, R. Moessner, and S. L. Sondhi, Nature (London) 451, 42 (2008).

[53] T.-H. Arima, J. Phys. Soc. Jpn. 82, 013705 (2013).
[54] P. Mendels, F. Bert, M. A. de Vries, A. Olariu, A. Harrison, F. Duc, J. C. Trombe, J. S. Lord, A. Amato, and C. Baines, Phys. Rev. Lett. 98, 077204 (2007).

[55] P. Dalmas de Réotier, A. Yaouanc, D. E. MacLaughlin, S. Zhao, T. Higo, S. Nakatsuji, Y. Nambu, C. Marin, G. Lapertot, A. Amato, and C. Baines, Phys. Rev. B 85, 140407 (2012). 